\title{
On the cosmological domain wall problem for the minimally extended supersymmetric standard model
}

\author{
S.A. Abel ${ }^{\text {a }}$, S. Sarkar ${ }^{\text {b, }, ~}$, P.L. White ${ }^{\mathrm{b}}$ \\ ${ }^{a}$ Rutherford Appleton Laboratory, Chilton, Didcot OXI1 OQX, UK \\ ${ }^{b}$ Theoretical Physics, University of Oxford, 1 Keble Road, Oxford OXI 3NP, UK
}

Received 28 June 1995; accepted 11 September 1995

\begin{abstract}
We study the cosmology of the Supersymmetric Standard Model augmented by a gauge singlet to solve the $\mu$-problem and describe the evolution of the domain walls which are created during electroweak symmetry breaking due to the discrete $\mathbb{Z}_{3}$ symmetry in this model. The usual assumption that (gravitationally induced) non-renormalisable terms which explicitly break this symmetry may cause the walls to collapse on a cosmologically safe timescale, is reconsidered. Such terms are constrained by considerations of primordial nucleosynthesis, and also because (by not respecting the $\mathbb{Z}_{3}$ symmetry) they induce divergences which destabilise the hierarchy and reintroduce the $\mu$-problem. We find that, even when the Kähler potential is 'non-minimal' (i.e. when the hidden sector couples directly to the visible), the model is either ruled out cosmologically or suffers from a naturalness problem.
\end{abstract}

\section{Introduction}

The purpose of introducing ( softly broken) supersymmetry into the Standard Model is to bring under control the quadratic divergences associated with a fundamental Higgs boson and make it 'natural' for its mass to be at the electroweak scale [1]. Yet the minimal supersymmetric Standard Model (MSSM) has its own naturalness problem. Its Lagrangian contains a term $\mu H_{1} H_{2}$ mixing the two Higgs doublets which are now required to give masses separately to the up- and down- type quarks. For successful

\footnotetext{
I PPARC Advanced Fellow.
} 
phenomenology $\mu$ should also be of order the electroweak scale but this must now be set by hand - the ' $\mu$-problem' $[2,3]$. To address this problem, the next-to-minimal supersymmetric Standard Model (NMSSM) [4] contains an additional singlet Higgs superfield $N$. By invoking a $\mathbb{Z}_{3}$ symmetry under which every chiral superfield $\Phi$ transforms as $\Phi \rightarrow \mathrm{e}^{2 \pi i / 3} \Phi$, the allowed terms in the superpotential are now $\lambda N H_{1} H_{2}-\frac{k}{3} N^{3}$ (in addition to the usual Yukawa terms generating fermion masses) while the Higgs part of the soft supersymmetry breaking potential is extended by the inclusion of two additional trilinear soft terms $A_{\lambda}$ and $A_{k}$ to

$$
\begin{aligned}
V_{\text {soft }}^{\text {Higgs }}= & -\lambda A_{\lambda}\left(N H_{1} H_{2}+\text { h.c. }\right)-\frac{k}{3} A_{k}\left(N^{3}+\text { h.c. }\right) \\
& +m_{H_{1}}^{2}\left|H_{1}\right|^{2}+m_{H_{2}}^{2}\left|H_{2}\right|^{2}+m_{N}^{2}|N|^{2},
\end{aligned}
$$

where $H_{1} H_{2}=H_{1}^{0} H_{2}^{0}-H^{-} H^{+}$. The $\mu$-term can now be simply set to zero by invoking the $\mathbb{Z}_{3}$ symmetry. An effective $\mu$-term of the form $\lambda\langle N\rangle$ will still be generated during $\mathrm{SU}(2)_{\mathrm{L}} \otimes \mathrm{U}(1)_{Y}$ breaking but it is straightforward to arrange that $\langle N\rangle$ is of order a soft supersymmetry breaking mass. Apart from solving the ' $\mu$-problem' the NMSSM also has interesting implications for supersymmetric phenomenology [5] and dark matter [6].

However, the NMSSM runs into a cosmological difficulty. The $\mathbb{Z}_{3}$ of the model is broken during the phase transition associated with electroweak symmetry breaking in the early universe. Due to the existence of causal horizons in an evolving universe, such spontaneously broken discrete symmetries lead to the formation of domains of different degenerate vacua separated by domain walls $[7,8]$. These have a surface energy density $\sigma \sim \nu^{3}$ where $\nu$ is a typical vacuum expectation value (vev) of the fields, here the electroweak scale of $\mathcal{O}\left(10^{2}\right) \mathrm{GeV}$. Such walls would come to dominate the energy density of the universe and create unacceptably large anisotropies in the cosmic microwave background radiation unless their energy scale is less than a few $\mathrm{MeV}$ [9]. Therefore cosmology requires the $\mathbb{Z}_{3}$ walls to disappear well before the present era. Following the original suggestion by Zel'dovich et al. [7], this may be achieved by breaking the degeneracy of the vacua, eventually leading to the dominance of the true vacuum. This happens when the pressure, i.e. the difference in energy density between the distinct vacua, begins to exceed the tension $\sigma / R$, where $\sigma$ is the surface energy density of the walls and $R$ the scale of their curvature. When $R$ becomes large enough for the pressure term to dominate, the domain corresponding to the true vacuum begins to expand into the domains of false vacuum and eventually fills all of space. It was recently argued [10] that gravitational interactions at the Planck scale $M_{\mathrm{PI}}$ would explicitly violate any discrete symmetry, causing just such a non-degeneracy in the minima of $\mathcal{O}\left(\nu^{5} / M_{\mathrm{Pl}}\right)$ where $\nu$ is a generic vev (of $\mathcal{O}\left(M_{W}\right)$ in our example). In fact, this suggestion had been applied already to the NMSSM in the context of string theories [11]. Thus there would appear to be a natural solution to the cosmological domain wall problem for the NMSSM.

In this paper we study whether this solution is indeed viable. In the following section we derive the structure of the walls, and show that the surface energy is approximately 
$M_{W}^{3}$ as expected on dimensional grounds. We go on to describe the evolution of the walls under the influence of the tension, the pressure due to the small explicit $\mathbb{Z}_{3}$ breaking and the friction due to particle reflections. In particular we demonstrate that wall domination of the energy density of the universe is avoided if the gravitationally induced terms are of order six or less. This is not however the tightest constraint on the domain walls; by applying constraints based on primordial nucleosynthesis we show that the magnitude of $\mathbb{Z}_{3}$ breaking must be $\gtrsim 10^{-7} \sigma M_{W}^{2} / M_{\mathrm{Pl}}$, in order to make the walls disappear before the nucleosynthesis era beginning at $T \sim 1 \mathrm{MeV}$. Thus only operators of dimension five (suppressed by at most one power of the Planck mass) are permitted. There are three such $\mathbb{Z}_{3}$ breaking terms which are allowed in the superpotential or Kähler potential and which induce dimension-5 operators in the effective potential. By inspection we find that the existence of one or more of these operators implies that there is no symmetry (discrete, global, gauged, $R$-symmetry or gauged $R$-symmetry) under which the lowenergy singlet can be charged. Consequently there cannot be any explanation for the absence of three additional allowed low energy operators which include the $\mu$-term itself as well as quadratic and linear terms in $N$. Thus our first conclusion is that not only does the NMSSM not solve the $\mu$-problem, it actually makes things worse by requiring the introduction of additional operators and by disallowing any symmetry which would forbid them.

We then go on to consider the fact that the singlet which appears in the NMSSM may introduce destabilising divergences [12]. Essentially the problem is that the introduction of non-renormalisable terms together with soft supersymmetry breaking produces corrections to the potential which are quadratically divergent and thus proportional to powers of the cut-off $\Lambda$ in the effective supergravity theory. Since the natural scale for this cut-off is $M_{\mathrm{Pl}}$, these can in principle destabilise the hierarchy, forcing the singlet vev and hence the scale of electroweak breaking to become very large (at least of order $\sqrt{M_{W} M_{\mathrm{Pl}}}$ ). By examining the possible $\mathbb{Z}_{3}$ breaking terms, we demonstrate that the removal of domain walls by this mechanism indeed destabilises the hierarchy. We conclude that the two constraints, viz. stability of the hierarchy and removal of domain walls, cannot be simultaneously satisfied by any gravitationally suppressed operators which one can add to the Lagrangian.

We consider alternative ways for dealing with the domain walls. One possible solution is to reintroduce the $\mu$ term in the superpotential in such a way as to avoid the introduction of the dangerous non-renormalisable operators. If we drop the assumption of minimality in the Kähler potential by allowing certain couplings of the hidden sector fields to the visible sector (as in Ref. [3]), we can retain $\mathbb{Z}_{3}$ symmetry in the full theory but break it spontaneously when supersymmetry is broken. In this way the hierarchy is not destabilised by tadpole diagrams. However the naturalness problem cannot be solved even for these more general models.

Finally we consider how gauge singlets may be accommodated in supersymmetry, without invoking these problems. There appear to be only a few possibilities, none of which yields a phenomenology bearing any resemblance to the NMSSM. 


\section{Domain walls in the NMSSM}

When a discrete symmetry is spontaneously broken as the universe expands and cools, 'domains' of the different degenerate vacua form, separated by narrow regions of higher potential called 'domain walls' [9]. The structure of these walls may be determined by finding time-independent solutions to the classical field equations after imposing the boundary conditions that at the endpoints the fields should be in distinct vacuum configurations. This has been done using numerical methods for the NMSSM potential [13] and we reiterate the essential features of the $\mathbb{Z}_{3}$ walls. As might be expected from dimensional arguments and by analogy with the analytically soluble case of a single real scalar field in a $\mathbb{Z}_{2}$ symmetric potential [9], the thickness and energy density of the walls are of order $\nu^{-1}$ and $\nu^{3}$ respectively, where $\nu$ is a typical vacuum expectation value. For naturalness reasons one would tend to assume that all three vacuum expectation values are of the same order; however, it is also possible that the singlet vev, $x$, is much larger than the usual $\nu=\sqrt{\nu_{1}^{2}+\nu_{2}^{2}}=174 \mathrm{GeV}$. This is in fact quite likely in the light of recent analyses where unification of soft terms and gauge couplings is imposed at the GUT scale; the only viable scenarios are then found to have $x / \nu \gtrsim 10$ with especially large values when the Higgs sector Yukawa couplings are very small [14]. In such cases, we would expect the wall to have a much higher surface energy $\sigma$; indeed we find that this is well approximated by

$$
\sigma \simeq 5 \times 10^{7} \mathrm{GeV}^{3}\left(\frac{k}{0.1}\right)\left(\frac{x}{5 \nu}\right)^{3},
$$

when $x$ is at least a few times larger than $\nu$. (This formula is accurate to about a factor of 2 in practice and is very good for large $x$, relative to both the trilinear soft terms and to $\nu$.) Similarly the thickness of walls is given by

$$
\delta \simeq 2 \times 10^{-2} \mathrm{GeV}^{-1}\left(\frac{k}{0.1}\right)^{-1}\left(\frac{x}{5 \nu}\right)^{-1}
$$

which again is most accurate when $x \gg \nu$ and $x \gg A_{k}, A_{\lambda}$. We show an example of a wall with large $x$ in Fig. 1. In comparison to the cases shown in Ref. [13], we see that the wall is thinner and the surface energy higher, as expected. (We note that if both $A_{k}$ and $A_{\lambda}$ are zero, then the $\mathbb{Z}_{3}$ symmetry of the scalar potential becomes a $U(1)$ symmetry, so the wall energy falls to zero and its width becomes infinite; in this limit however we have an axion problem. We find that if $A_{k}$ or $A_{\lambda}$ are greater than a few $\mathrm{GeV}$ then the wall energy is insensitive to their exact values.)

Immediately after the electroweak phase transition the universe is filled with equal volumes of the three degenerate phases. These are correlated on a length scale which depend on the nature of the phase transition, varying from $\xi \sim T_{\mathrm{c}}^{-1}$ for a second-order transition to $\xi \sim H^{-1}$ for a strongly first-order transition $[9,15]$. Since the probability for each vacuum $(0.333)$ is just above the percolation threshold (which for continuum percolation theories is found to be 0.295 [16]), the universe is then filled with highly convoluted, infinite regions separated by stable domain walls of typical curvature scale $\xi$, which rapidly grows to the size of the horizon. 

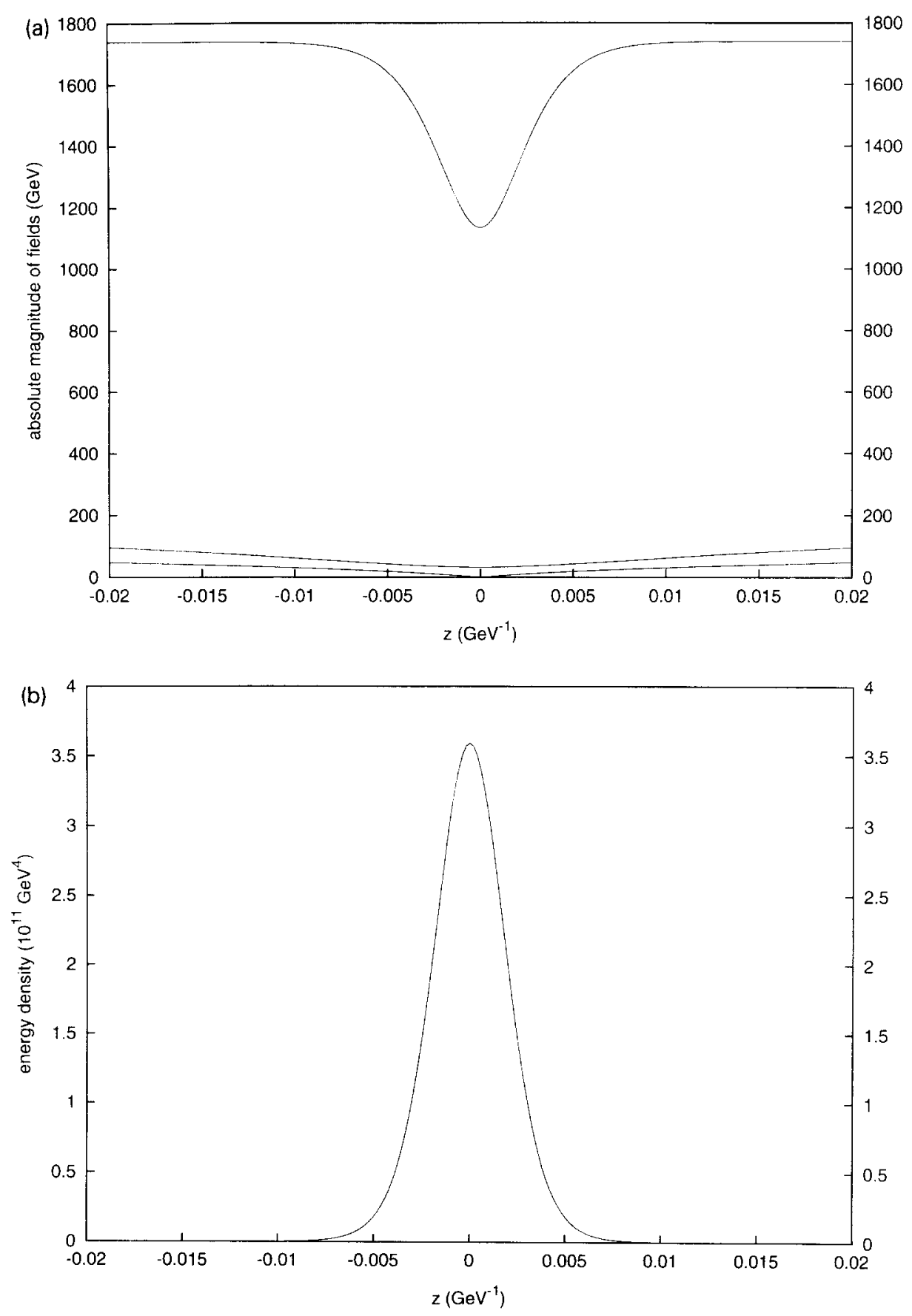

Fig. 1. (a), (b) An example of a wall configuration with the singlet vev $x=10 \nu$. Here we have chosen $\tan \beta=2, \lambda=k=0.2, A_{k}=A_{\lambda}=200 \mathrm{GeV}$. The total surface energy density is $8.6 \times 10^{8} \mathrm{GeV}^{3}$. Fig. $1 \mathrm{a}$ shows the values of the three scalar fields as a function of position while Fig. lb shows the energy density in the wall relative to the vacuum. 
Let us now turn to the dynamics of cosmological networks of such walls. As discussed in Ref. [7], the most important forces acting on the walls are surface tension, friction and pressure. The equation of motion for a quasi-spherical piece of wall moving with velocity $\dot{R}$ (with $\gamma \equiv 1 / \sqrt{1-\dot{R}^{2}}$ ) and having local radius of curvature $R$, is

$$
\frac{\mathrm{d}^{2} R}{\mathrm{~d} t^{2}}=-\frac{2}{R \gamma^{2}}-\frac{\langle n p v\rangle}{\sigma \gamma^{3}}-\frac{\varepsilon}{\sigma \gamma^{3}} .
$$

The first term on the RHS reflects the fact that it is energetically favourable for the wall network to reduce its surface area through surface tension, and hence small domains will collapse, irregularities in the surfaces will straighten out, and the correlation length will increase. This term expresses just the conservation of energy in the absence of pressure and friction.

The second term on the RHS corresponds to friction arising due to the interactions of the wall network with the thermal plasma. As particles reflect off the walls, they exert a force given by the thermally averaged momentum transfer $\langle n p v\rangle$, where $n$ is the particle density, $v$ the particle velocity relative to the wall, and $p$ the momentum perpendicular to the wall. (Actually the friction is $\propto v$ only when $v \ll c$.) Friction is clearly important at times very close to the electroweak phase transition if the top quark and gauge boson fields are still in equilibrium in the plasma. At later times, when the number density of these particles is exponentially suppressed, the main source of friction is the interaction of the walls with lighter fermions in the plasma. The constant difference in phase in the mass terms on either side of the wall (i.e. $\pi / 3$ or $2 \pi / 3$ ) does not by itself cause any reflection but rather just a phase shift in the fermion masses (as can be checked by equating transmission and reflection coefficients at the wall). In order to estimate the reflection coefficient, it is useful to describe the space dependent mass by the inverted bell-shaped function

$$
m^{2}\left(x_{\perp}\right)=m^{2}-a^{2} \frac{\lambda(\lambda-1)}{\cosh ^{2} a x_{\perp}}
$$

where $x_{\perp}$ is the perpendicular distance from the wall, and $m$ is the mass given to the reflecting particle by the Higgs fields which comprise the domain wall of width $a^{-1}$. The task of finding the reflection coefficient (using the Klein-Gordon equation) then reduces to a known problem, the modified Pöschl-Teller potential, which can be solved analytically (see for example Ref. [17]). We take the depth of the well to be $m^{2}$ and the width $a^{-1} \sim M_{W}$. The depth parameter is $\lambda=\left(1+m^{2} / M_{W}^{2}\right)$ and the reflection coefficient is then found to be

$$
|R|^{2}=\frac{\pi^{2} m^{4}}{\pi^{2} m^{4}+M_{W}^{4} \sinh ^{2} \pi p a} \approx \frac{m^{4}}{p^{2} M_{W}^{2}},
$$

where we have taken $M_{W} \gg p \gg m$ as is appropriate once the gauge bosons and top quark have fallen out of equilibrium. (There is a region at low energy $|p|<m^{2} / M_{W}$ in which the particles experience total reflection [9]. However this contribution is insignificant here, being suppressed by many powers of $m / M_{W}$.) Clearly particles which are 


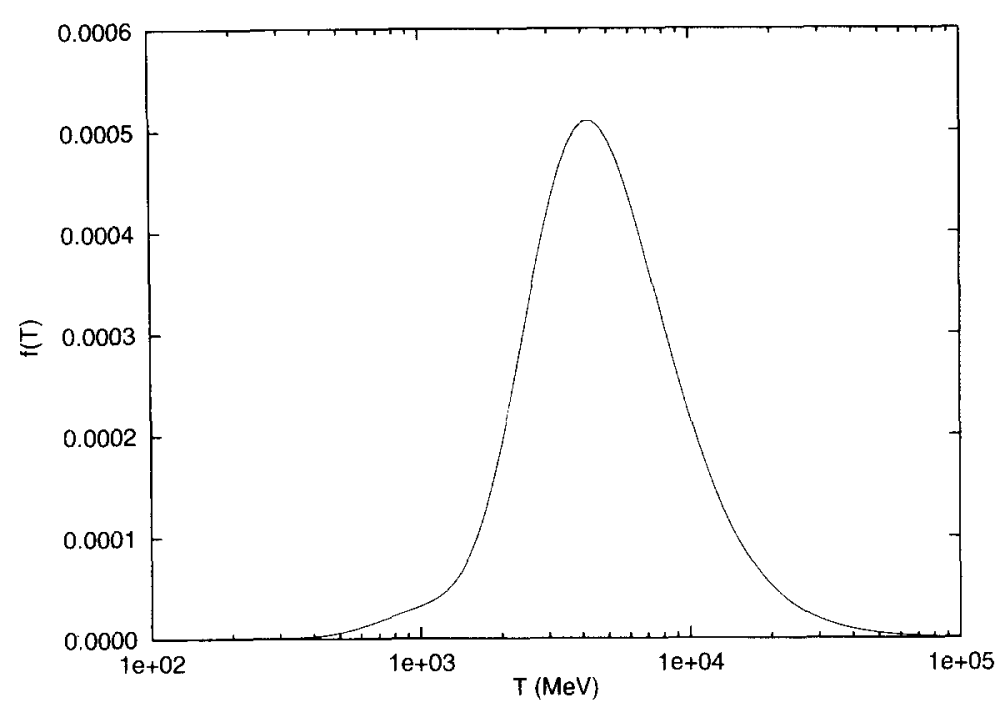

Fig. 2. The function $f(T)$ [see Eq. (12)] related to friction.

heavy, especially the bottom quark, will be more important here. We can estimate the friction by considering a particle of mass $m$, when the wall velocity through the plasma, $u$, is small. Then

$$
\langle n p v\rangle=g \int \frac{\mathrm{d}^{3} p}{(2 \pi)^{3}} \frac{m^{4}}{p^{2} M_{W}^{2}} \frac{p_{\perp}^{2}}{E}\left[\frac{1}{\mathrm{e}^{\left(\gamma E+\gamma u p_{\perp}\right) / T}+1}-\frac{1}{\mathrm{e}^{\left(\gamma E-\gamma u p_{\perp}\right) / T}+1}\right],
$$

where $T$ is the temperature of the plasma, and $g$ is the number of degrees of freedom of the reflecting particles. Expanding this function in $u$ keeping the leading term only and performing the angular integral, we find

$$
\langle n p v\rangle=\frac{g u}{8 \pi^{2}} T^{4} \frac{T^{2}}{M_{W}^{2}} F\left(x_{m}\right),
$$

where

$$
F\left(x_{m}\right)=\int_{x_{m}}^{\infty} \mathrm{d} x x_{m}^{4}\left(x^{2}-x_{m}^{2}\right) \frac{\mathrm{e}^{x}}{\left(\mathrm{e}^{x}+1\right)^{2}},
$$

and we have defined $x \equiv E \gamma / T$ and $x_{m} \equiv m \gamma / T$. This integral is very well approximated by

$$
F\left(x_{m}\right)=x_{m}^{5}\left(0.6 \mathrm{e}^{-x_{m}}\right)^{3} .
$$

Summing over all the particle species in the plasma, we find that

$$
\langle n p v\rangle=f(T) u \frac{T^{4}}{8 \pi^{2}},
$$

where $f(T)<5 \times 10^{-4}$ at all temperatures. We show $f(T)$ in Fig. 2 where, apart from omitting the contribution of the up and down quarks, we have neglected the possible 
effect of the quark-hadron phase transition. In the era when pressure is negligible (i.e. when the typical curvature scale is small), we can calculate the terminal wall velocity, $u_{\text {term }}$, and establish a posteriori that our approximation of small $u$ to obtain Eq. (8) is indeed correct, i.e. friction is important. Substituting the friction into Eq. (4), we find

$$
u_{\mathrm{term}}=\frac{16 \pi^{2}}{f(T)}\left(\frac{\sigma}{T^{4} R}\right) .
$$

For typical values of the radius, $R \sim u_{\text {term }} t$, we see that friction is important only at temperatures above a few hundred $\mathrm{MeV}$. We therefore conclude that shortly after the quark-hadron phase transition the walls move with velocities comparable to the speed of light and so we may safely neglect friction in what follows.

The last term on the RHS in Eq. (4) is the pressure corresponding to the difference $\varepsilon$ in the energy density between the different vacua. As remarked earlier, this will become dominant when it exceeds the surface tension, i.e. when

$$
\varepsilon>\frac{\sigma}{R}
$$

We show this happening in Fig. 3, where we have performed a simple thin wall simulation of a network of domain walls using techniques similar to those used in Ref. [18], and which we have discussed in more detail elsewhere [13]. In the absence of friction it is convenient to rescale the parameters with some typical length scale, $R_{0}$, which we choose to be $1 \mathrm{~cm}$, corresponding approximately to the curvature scale when pressure becomes dominant if $\varepsilon \sim M_{W}^{5} / M_{\mathrm{Pl}}$. Thus defining $\rho \equiv R / R_{0}$, and $\tau \equiv t / R_{0}$, Eq. (4) becomes

$$
\frac{\mathrm{d}^{2} \rho}{\mathrm{d} \tau^{2}}=-\frac{2}{\rho \gamma^{2}}-\frac{\varepsilon R_{0}}{\gamma^{3} \sigma} .
$$

Thus there are only two independent parameters in our simulation, given by the pressure in each of the two false vacua, $\varepsilon R_{0} / \sigma$. Initially, the walls expand under their own tension, and the structure develops in the manner discussed in Refs. [18,19]. Eventually pressure dominates as expected and the entire volume is cleared of walls. This contrasts with the no-pressure case, where one or two horizon-sized walls always remain [13]. The behaviour for different values of the pressure or surface tension is identical if the time and length, respectively, are scaled appropriately.

One might consider the possibility that since frictionless, pressureless walls expand until there is roughly one wall per horizon scale [9], domain walls may be accommodated by simply assuming that our local region of space-time just happens to be empty of them, i.e. that there is a wall lurking just outside our present horizon. There are at least two objections to this. Firstly the walls eventually come to dominate the energy density of the universe, causing unacceptable 'power-law' inflation [20], unless their separation is many times greater than the present horizon scale, which is clearly impossible by causality. Secondly, even such a wall outside the horizon will have a curvature scale comparable to the present horizon scale and thus induce unacceptably large anisotropy in the cosmic microwave background [21]. 


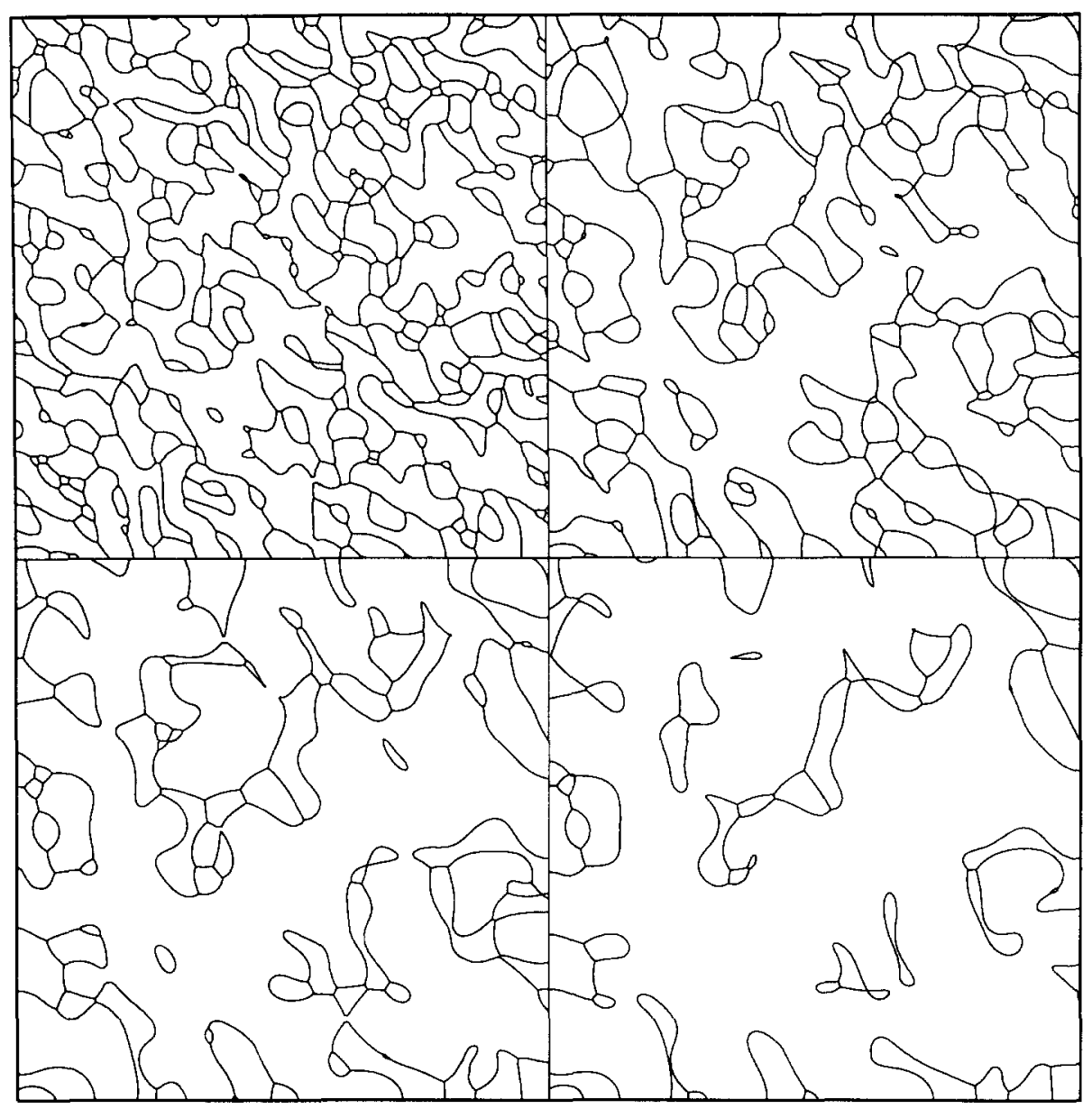

Fig. 3. A typical example of the evolution of the wall network with a pressure term of order $\sigma M_{W}^{2} / M_{\mathrm{Pl}}$. The figure shows the wall network at four epochs separated by an interval of $10^{-10} \mathrm{sec}$, beginning at the time when pressure starts to dominate the evolution.

\section{When walls collide}

What value of the pressure (i.e. explicit $\mathbb{Z}_{3}$ breaking) is required to safely remove the walls? The crudest estimate we can make is simply to insist that the walls are removed before they dominate over the radiation energy density in the universe, in order to avoid wall driven inflation. Since the walls move at close to the speed of light below the quark hadron phase transition, their curvature scale will be roughly the horizon size, $R \sim t \sim M_{\mathrm{Pl}} / g_{*}^{1 / 2} T^{2}$. Since the energy density of the walls is

$$
\rho_{\text {walls }} \sim \frac{\sigma}{R},
$$


and the radiation energy density is $\rho_{\text {rad }} \sim g_{*}^{1 / 2} T^{4}$, we see that walls dominate the evolution below a temperature

$$
T_{\star} \sim\left(\frac{\sigma}{g_{*}^{1 / 2} M_{\mathrm{Pl}}}\right)^{1 / 2}
$$

To prevent this we require the pressure to have become dominant before this epoch, i.e.

$$
\varepsilon>\frac{\sigma}{R_{\star}} \sim \frac{\sigma^{2}}{M_{\mathrm{Pl}}^{2}} .
$$

A pressure of this magnitude would be produced by dimension- 6 operators in the potential. However, one should consider further constraints coming from primordial nucleosynthesis, and we find that only operators of dimension five or less are sufficient to satisfy these. In fact for weak-scale walls the time associated with the temperature $T_{\star}$ is $t_{\star} \sim M_{\mathrm{Pl}}^{2} / g_{*}^{1 / 2} M_{W}^{3} \sim 10^{8} \mathrm{sec}$, i.e. long after nucleosynthesis. The entropy produced when the walls collide (which is by now a major proportion of the total entropy in the universe) is dumped into all the decay products of neutral Higgs particles, i.e. Standard Model quarks and leptons. In order to check whether this violates phenomenological bounds, we compute the relative energy density released in such collisions, viz.

$$
\begin{aligned}
\frac{\rho_{\text {walls }}}{n_{\gamma}} & \sim \frac{\sigma}{t n_{\gamma}} \\
& \sim 7 \times 10^{-11} \mathrm{GeV}\left(\frac{\sigma}{M_{W}^{3}}\right)\left(\frac{t}{\mathrm{sec}}\right)^{1 / 2},
\end{aligned}
$$

where we have taken the number of relativistic degrees of freedom in the plasma to be $g_{*}=43 / 4$. Detailed consideration of the effects of high energy particles on primordial nucleosynthesis and on the $2.73 \mathrm{~K}$ Planckian spectrum of the microwave background radiation impose severe upper limits on this parameter [22]. For the typical values of $\sigma$ in Fig. 1, we find that the walls are required to disappear before the onset of nucleosynthesis at about $0.1 \mathrm{sec}$, as otherwise the hadrons in the showers triggered by the decay products would alter the neutron-to-proton ratio, resulting in a ${ }^{4} \mathrm{He}$ mass fraction in excess of the conservative observational upper bound of $25 \%$ [23]. This means that in order not to disrupt primordial nucleosynthesis, we require explicit $\mathbb{Z}_{3}$ breaking of magnitude

$$
\varepsilon \sim \lambda^{\prime} \sigma M_{W}^{2} / M_{\mathrm{Pl}}
$$

with

$$
\lambda^{\prime} \geq 10^{-7}
$$

\section{The return of the $\mu$ problem}

Having established that one needs dimension $-5, \mathbb{Z}_{3}$ breaking operators to appear in the effective potential, we can consider ways in which this can be achieved by adding terms 
to the Kähler potential or superpotential. We first assume that these are 'minimal' in the sense that they do not contain couplings between the hidden and visible sectors (which couple only through gravity). Later we shall consider the most general non-minimal case. In all cases we find that there is a naturalness problem associated with the explicit breaking of the $\mathbb{Z}_{3}$ symmetry.

Let us write down the contributions to the supergravity Lagrangian which explicitly break $\mathbb{Z}_{3}$, and which are invariant under the NMSSM gauge group. These are

$$
\lambda^{\prime} \frac{N^{4}}{M_{\mathrm{Pl}}}, \quad \lambda^{\prime} \frac{N^{2}\left(H_{1} H_{2}\right)}{M_{\mathrm{Pl}}}, \quad \lambda^{\prime} \frac{\left(H_{1} H_{2}\right)^{2}}{M_{\mathrm{Pl}}},
$$

in the superpotential, and

$$
\alpha_{i} \frac{\left(N+N^{\dagger}\right)\left(H_{i} H^{i \dagger}\right)}{M_{\mathrm{Pl}}}, \quad \beta\left(\frac{N^{\dagger} H_{1} H_{2}+\text { h.c. }}{M_{\mathrm{Pl}}}\right),
$$

in the Kähler potential. As in Ref. [12], we can absorb the last two contributions into the superpotential to $\mathcal{O}\left(M_{\mathrm{Pl}}^{-1}\right)$ by making the redefinitions

$$
\begin{gathered}
H_{i} \rightarrow\left(1-\frac{\alpha_{i} N}{M_{\mathrm{PI}}}\right) H_{i}, \\
N \rightarrow N-\frac{\beta\left(H_{1} H_{2}\right)}{M_{\mathrm{PI}}},
\end{gathered}
$$

and so we shall consider only the first three contributions in what follows. Inspecting these, we observe that $N$ must be a singlet under any additional symmetry in order for any one of these terms to exist in addition to the terms $N^{3}$ and $N H_{1} H_{2}$ in the low energy superpotential. In other words, each of them implies that the following 'unnatural' contribution to the superpotential is invariant

$$
\delta W_{\text {'unnatural' }}=\mu^{\prime \prime} N+\mu^{\prime} N^{2}+\mu H_{1} H_{2} .
$$

Thus not only have we reintroduced the $\mu$-problem, we now have two additional naturalness problems. Whereas the standard $\mu$-problem may well be solved at a future date (for example by the mechanism of Ref. [3]), we shall see that the naturalness problem which has reappeared here can have no solution based on an underlying symmetry.

\section{The return of the hierarchy problem}

As if the difficulties above were not bad enough, there is the possibility of quadratic tadpole divergences which can lead to a destabilisation of the hierarchy [12]. This exacerbates our problems, since such divergences arise at each order in perturbation theory, forcing us to re-fine-tune. These are a potential problem in any supergravity model with gauge singlets since the dangerous diagrams are not excluded by gauge invariance. 

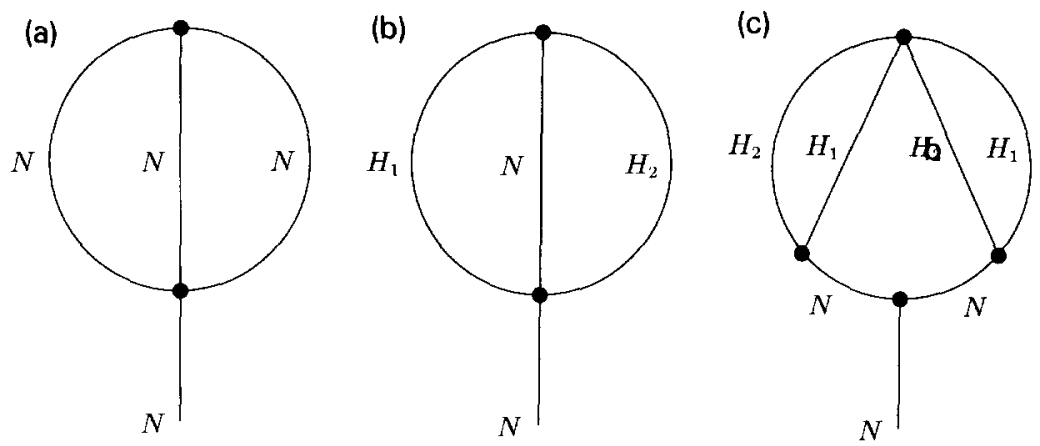

Fig. 4. (a), (b), (c) The three dangerous diagrams for each of the three operators which can destabilise the hierarchy.

These diagrams arise when supersymmetry is spontaneously broken, because superWeyl-Kähler invariance necessitates that the vev of the Kähler potential become nontrivial. In fact [12]

$$
\begin{aligned}
\left\langle\mathrm{e}^{2 K / 3}\right\rangle & \approx \mathrm{e}^{2 K / 3} \mid\left(1+\theta^{2} M_{\mathrm{s}}^{2}+\bar{\theta}^{2} M_{\mathrm{s}}^{2}+\theta^{2} \bar{\theta}^{2} M_{\mathrm{s}}^{4}\right), \\
\langle\phi\rangle & \approx \phi \mid\left(1+\theta^{2} M_{\mathrm{s}}^{2}\right),
\end{aligned}
$$

where $\phi$ is the chiral compensator, $M_{\mathrm{s}}$ is the scale of supersymmetry breaking in the hidden sector, and the RHS refers to only the scalar components. The leading tadpole divergences are quadratic and appear at two-loop order for the first two operators in Eq. (21). In our case, the diagrams responsible are shown in Figs. $4 \mathrm{a}$ and $4 \mathrm{~b}$, and they lead to the terms

$$
\frac{\lambda^{\prime} k}{3\left(16 \pi^{2}\right)^{2}}\left(\phi_{N}+\phi_{N}^{*}\right) M_{\mathrm{P} 1} m_{3 / 2}^{2}+\frac{\lambda^{\prime} k}{3\left(16 \pi^{2}\right)^{2}}\left(F_{N}+F_{N}^{*}\right) M_{\mathrm{P} 1} m_{3 / 2}
$$

and

$$
\frac{\lambda^{\prime} \lambda}{\left(16 \pi^{2}\right)^{2}}\left(\phi_{N}+\phi_{N}^{*}\right) M_{\mathrm{P} 1} m_{3 / 2}^{2}+\frac{\lambda^{\prime} \lambda}{\left(16 \pi^{2}\right)^{2}}\left(F_{N}+F_{N}^{*}\right) M_{\mathrm{P} 1} m_{3 / 2}
$$

respectively, where we have taken the cut-off to be $\Lambda \sim M_{\mathrm{Pl}}$ and introduced the

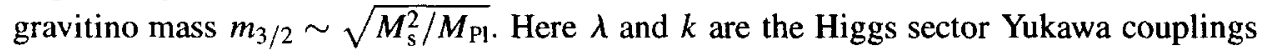
defined earlier. The third term in Eq. (21) gives rise to a divergence at three-loop order as shown in Fig. $4 \mathrm{c}$ and the calculation is a little more tricky. Using the perturbation theory rules of Ref. [12], quadratic divergences are indeed found to arise of the form

$$
\frac{\lambda^{\prime} \lambda^{2} k}{\left(16 \pi^{2}\right)^{3}}\left(\phi_{N}+\phi_{N}^{*}\right) M_{\mathrm{P} 1} m_{3 / 2}^{2}
$$

and

$$
\frac{\lambda^{\prime} \lambda^{2} k}{\left(16 \pi^{2}\right)^{3}}\left(F_{N}+F_{N}^{*}\right) M_{\mathrm{P} \mid} m_{3 / 2},
$$


where we have replaced a quadratically divergent three loop integral with a cut-off, $M_{\mathrm{Pl}}^{2}$. All of these terms naturally drive the vev of the singlet (and hence of $H_{1}, H_{2}$ ) to the hidden sector scale, $\langle x\rangle \approx \sqrt{m_{3 / 2} M_{\mathrm{Pl}}} \sim 10^{11} \mathrm{GeV}$. If we wish to avoid the reappearance of the hierarchy problem, these terms should be smaller than $\sim\left(\phi_{N}+\phi_{N}^{*}\right) m_{3 / 2}^{3}$ or $\sim$ $\left(F_{N}+F_{N}^{*}\right) m_{3 / 2}^{2}$. Even for the three loop diagram this requires

$$
\lambda^{\prime} \lesssim 3 \times 10^{-11},
$$

where we have taken $m_{3 / 2} \sim M_{W}$. Clearly this bound is only approximate, since we do not know the precise values of the Yukawa couplings $\lambda$ and $k$, which we have taken here to be of $\mathcal{O}(1)$. However, it should also be borne in mind that one would like to have control over the scale of electroweak symmetry breaking. That is we do not wish the mass of the $W$ to depend strongly on the (unknown) physics at the Planck scale, i.e. on $\lambda^{\prime}$. In order to achieve this, the above bound should be tightened even further.

The bound in Eq. (30) is clearly incompatible with that in Eq. (20) required for successful nucleosynthesis, and we conclude that the NMSSM, at least in the models with 'minimal' Kähler potentials, has either a domain wall problem or a hierarchy problem.

\section{A solution to the hierarchy problem}

Is it possible that we can solve these problems by allowing the hidden and visible sectors to mix? In this section we shall see that the answer is yes for the destabilising divergences, but no for the naturalness problem. In other words, we are able to regain perturbative control over the scale of electroweak symmetry breaking, but we find, quite generally, that certain couplings must be set by hand initially to be small. This leads to a naturalness problem of at least one part in $10^{9}$.

In order to eliminate destabilising divergences, we must drop our insistence on minimality in the Kähler potential, by allowing the hidden and visible sectors to mix. In this case, models similar to the NMSSM can be constructed. We use a mechanism similar to that in Ref. [3], and find that modells with (Standard Model) singlets can have naturally large $N^{2}, N^{3}$ and $\mu$ terms.

The Giudice-Masiero mechanism [3] seeks to solve the $\mu$ problem for the MSSM by generating it via the Kähler potential. That is we have

$$
\mathcal{G}=y^{i} y_{i}^{\dagger}+z z^{\dagger}+\left(\frac{\alpha}{M_{\mathrm{Pl}}} z^{\dagger} H_{1} H_{2}+\text { h.c. }\right)+M_{\mathrm{Pl}}^{2} \ln \left|\frac{f(z)+g(y)}{M_{\mathrm{P} 1}^{3}}\right|^{2}
$$

where the $y_{i}$ fields belong to the visible sector, and the $z$ singlet field belongs to the hidden sector. $\mathcal{G}$ is Kähler invariant. The label 'hidden' is justified when we take the "flat" limit $M_{\mathrm{Pl}} \rightarrow \infty$ in the effective potential (keeping $M_{s}^{2} / M_{\mathrm{Pl}}$ fixed), and find that the $z$ field, which acquires a vev of $\mathcal{O}\left(M_{\mathrm{Pl}}\right)$, decouples from the visible sector, apart from inducing soft supersymmetry breaking terms and a $\mu$ term proportional to $\alpha$, via gravitational couplings. These are all of $\mathcal{O}\left(M_{\mathrm{s}}^{2} / M_{\mathrm{Pl}}\right)$, where $M_{\mathrm{s}}$ is the aforementioned 
scale of supersymmetry breaking in the hidden sector which we introduce by hand. Having introduced a new coupling between the visible and hidden sectors, we must invoke some symmetry which forbids other couplings as well as a coupling $\mathrm{M}_{\mathrm{Pl}} \mathrm{H}_{1} \mathrm{H}_{2}$ in the superpotential. This could be a Peccei-Quinn symmetry, a discrete symmetry, or a gauged or global $R$ symmetry. In addition the presence of a new symmetry rules out the simplest version of the Polonyi model (which in view of its severe cosmological problems [24] might not be such a bad thing).

For the next-to-minimal choice of Kähler potential above, the terms in the scalar potential are

$$
V_{\text {scalar }}=\hat{g}_{i} \hat{g}^{i}+m_{3 / 2}^{2} y_{i} y^{i}+m^{\dagger}\left[y^{i} g_{i}+(A-3) \hat{g}^{(3)}+(B-2) m_{3 / 2} \mu H_{1} H_{2}+\text { h.c. }\right],
$$

where $\hat{g}^{(3)}$ are the trilinear terms of the superpotential, rescaled according to

$$
\hat{g}^{(3)}=\left\langle\exp \left(z z^{\dagger} / 2 M_{\mathrm{Pl}}^{2}\right)\right\rangle g^{(3)} .
$$

Here $\hat{g}$ is the new low energy superpotential including the $\mu$ term

$$
\hat{g}=\hat{g}^{(3)}+\mu H_{1} H_{2},
$$

and $m_{3 / 2}$ is the gravitino mass

$$
m_{3 / 2}=\left\langle\exp \left(z z^{\dagger} / 2 M_{\mathrm{Pl}}^{2}\right) f^{(2)}\right\rangle,
$$

where the vev of $f^{(2)}=M_{\mathrm{s}}^{2} / M_{\mathrm{Pl}}$ is set by hand such that $M_{\mathrm{s}} \sim 10^{11} \mathrm{GeV}$. The $\mu$ term is given by

$$
|\mu|=\left|\alpha m\left\langle\frac{M_{\mathrm{Pl}} f_{z}}{f}\right\rangle\right| \text {. }
$$

Applying the constraint of vanishing cosmological constant, the authors of Ref. [3] found

$$
\begin{aligned}
B & =(2 A-3) /(A-3), \\
|\mu| & =|m \alpha(A-3) / \sqrt{3}|,
\end{aligned}
$$

where $A$ is the universal trilinear scalar coupling, $A=\sqrt{3}\left\langle z / M_{\mathrm{Pl}}\right\rangle$. Now let us apply the same mechanism to a model with MSSM singlets, $N$. The most obvious extension is to choose the Kähler potential

$$
\mathcal{G}=y^{i} y_{i}^{\dagger}+z z^{\dagger}+\left(\frac{\alpha}{M_{\mathrm{Pl}}} z^{\dagger} H_{1} H_{2}+\frac{\alpha^{\prime}}{M_{\mathrm{Pl}}} z^{\dagger} N^{2}+\text { h.c. }\right)+M_{\mathrm{Pl}}^{2} \ln \left|\frac{f(z)+g(y)}{M_{\mathrm{Pl}}^{3}}\right|^{2},
$$

where, in this case, $f(y)$ is the superpotential of the NMSSM. The hidden sector field, $z$, has the opposite charge to $N$ under the $\mathbb{Z}_{3}$ symmetry so that the full theory is $\mathbb{Z}_{3}$ invariant. In this case $\mathbb{Z}_{3}$ is broken spontaneously at the Planck scale and the resulting 
domain walls are presumably removed during inflation. The low energy scalar potential is

$$
\begin{gathered}
V_{\text {scalar }}=\hat{g}_{i} \hat{g}^{i}+m^{2} y_{i} y^{i}+m^{\dagger}\left[y^{i} g_{i}+(A-3) \hat{g}^{(3)}+(B-2) m \mu H_{1} H_{2}\right. \\
\left.+(B-2) m \mu^{\prime} N^{2}+\text { h.c. }\right]
\end{gathered}
$$

where

$$
\begin{aligned}
\hat{g} & =\hat{g}^{(3)}+\mu H_{1} H_{2}+\mu^{\prime} N^{2} \\
|\mu| & =\left|\frac{m \alpha(A-3)}{\sqrt{3}}\right|, \quad\left|\mu^{\prime}\right|=\left|\frac{m \alpha^{\prime}(A-3)}{\sqrt{3}}\right| .
\end{aligned}
$$

Notice that the low energy model has generally far more terms in its low energy lagrangian than the NMSSM. The latter (and the $\mathbb{Z}_{3}$ symmetry) is in fact recovered when we let $\alpha=\alpha^{\prime}=0$; thus we can break the $\mathbb{Z}_{3}$ symmetry by as much or as little as we like.

Although this model has removed the problem of destabilising divergences (it is now no longer possible to write down any of the divergent tadpole diagrams), it does not quite solve the naturalness problem (i.e. the presence of small couplings unprotected by any symmetry), since there is still the coupling $z N$ which is allowed under the $\mathbb{Z}_{3}$ symmetry, and which no other symmetry can forbid. These may be set to zero by hand and will stay zero by virtue of the nonrenormalisation theorem.

One might wonder if by somehow extending the Kähler potential it may be possible to exclude these terms. As we now show however, this is not the case, and no matter how complicated we make the Lagrangian, the naturalness problem associated with the absence of the $z N$ couplings stays with us. Consider the most general supergravity Lagrangian, in which the only requirement we make is that the superpotential contains the terms

$$
\delta g(y)=\frac{k^{a b c}(\xi)}{3 ! 3} N_{a} N_{b} N_{c}+\lambda^{a b c}(\xi)\left(H_{1} H_{2}\right)_{a b} N_{c},
$$

where $a, b, c$ are indices representing some symmetry group (discrete or otherwise), and the couplings are holomorphic function of the hidden sector fields, $\xi_{a}=z_{a} / M_{\mathrm{Pl}}$. The breaking of $\mathbb{Z}_{3}$ symmetry in the visible sector by operators of dimension five, requires that we also include at least one of the operators,

$$
\begin{aligned}
& \Lambda^{a b}(\xi, \bar{\xi}) N_{a} N_{b} \\
& \Lambda^{a b}(\xi, \bar{\xi})\left(H_{1} H_{2}\right)_{a b} \\
& \Lambda_{c}^{a b}(\xi, \bar{\xi}) N_{a} N_{b} N^{\dagger c} \\
& \Lambda_{c}^{a b}(\xi, \bar{\xi})\left(H_{1} H_{2}\right)_{a b} N^{\dagger c} \\
& \Lambda^{a b c d}(\xi) N_{a} N_{b} N_{c} N_{d} \\
& \Lambda^{a b c d}(\xi) N_{a} N_{b}\left(H_{1} H_{2}\right)_{c d} \\
& \Lambda^{a b c d}(\xi)\left(H_{1} H_{2}\right)_{a b}\left(H_{1} H_{2}\right)_{c d},
\end{aligned}
$$


where the first four operators give dimension-5 operators if they appear in the Kähler potential or superpotential, but the last three operators must appear in the superpotential, hence their couplings are holomorphic functions of the hidden sector fields. If we make the assumption that the couplings $k^{a b c}$ and $\lambda^{a b c}$ are invertible, then corresponding to each of the operators above, there is an additional invariant operator which is some function of the hidden sector fields multiplied by $N_{a}$. These are, respectively,

$$
\begin{aligned}
& \Lambda_{a b}^{\dagger}\left(k^{-1}\right)^{\dagger a b c} N_{c} \\
& \Lambda_{a b}^{\dagger}\left(\lambda^{-1}\right)^{\dagger a b c} N_{c} \\
& \Lambda_{c}^{a b}\left(k^{-1}\right)_{a b d}\left(k^{-1}\right)^{\dagger c d e} N_{e} \\
& \Lambda_{c}^{a b}\left(\lambda^{-1}\right)_{a b d}\left(k^{-1}\right)^{\dagger c d e} N_{e} \\
& \Lambda^{a b c d}\left(k^{-1}\right)_{a b e}\left(k^{-1}\right)_{c d f}\left(k^{-1}\right)^{\dagger e f g} N_{g} \\
& \Lambda^{a b c d}\left(k^{-1}\right)_{a b e}\left(\lambda^{-1}\right)_{c d f}\left(k^{-1}\right)^{\dagger e f g} N_{g} \\
& \Lambda^{a b c d}\left(\lambda^{-1}\right)_{a b e}\left(\lambda^{-1}\right)_{c d f}\left(k^{-1}\right)^{\dagger e f g} N_{g} .
\end{aligned}
$$

The least damage to the effective potential occurs if these terms appear in the Kähler potential, in which case we find terms of the form

$$
m_{3 / 2}^{2} M_{\mathrm{Pl}} \phi_{N}+\text { h.c. }
$$

appearing in the effective potential. Thus the natural scale of the singlet vev is $\sim 10^{11}$ $\mathrm{GeV}$ and since it should be less than the electroweak scale, this constitutes a naturalness problem of at least one part in $10^{9}$.

\section{Conclusions}

Before concluding, we should mention a few escape clauses, none of which however are very appealing:

(i) The most obvious is to introduce the $\mu$ term into the superpotential by the GiudiceMasiero mechanism [3] and simply set to zero all of the operators which might give $N$ a large vev. (Although this appears to be rather unaesthetic, one might remark that the naturalness problem which results is no worse than that already with us due to the smallness of $M_{\mathrm{S}}$ compared to the Planck mass. Since the "unnaturalness" is of the same order, it may even be possible to construct the Kähler potential so that the two naturalness problems are connected.)

(ii) Alternatively one can invoke inflation at the weak scale to remove all the domain walls, just as has been suggested in the context of other unwanted relics, e.g. string moduli [25]. However such a scenario must be very finely tuned - the domain walls must be adequately diluted without erasing the density perturbations generated by inflation at the GUT scale [26]). (Although density perturbations are also generated during weak scale inflation, the small value of the Hubble parameter would make these too small to account for the microwave background anisotropies observed by COBE.) Sec- 
ondly, the reheat temperature must be high enough for both successful baryogenesis and nucleosynthesis. We are not aware of any likely candidate for the required scalar field.

(iii) The $\mathbb{Z}_{3}$ symmetry could be broken at a high scale, $M_{\text {contrived, in the visible sector }}$ and explicit $\mathbb{Z}_{3}$ breaking terms induced. This is similar to the solution to the hierarchy problem discussed earlier, with the "advantage" that the fine tuning is driven by $M_{\text {contrived }}$ rather than $M_{\mathrm{P} 1}$. However this will still entail a fine tuning of approximately one part in $10^{12}$, since in order for the walls to be inflated away, $M_{\text {contrived }}$ should exceed the inflationary scale of $\sim 10^{14} \mathrm{GeV}$ as deduced from normalisation to the COBE data [26]. Otherwise one would have to invoke a second epoch of inflation at an intermediate scale, with its own attendant problems (see above).

(iv) The $\mathbb{Z}_{3}$ symmetry could be made anomalous by adding extra fields to the theory which couple to $\mathrm{SU}(3)_{c}$ (for example an additional generation). In this case the symmetry is broken non-perturbatively at the quark-hadron phase transition, and the walls collapse very soon thereafter [27]. However, it is difficult to see how this constitutes a solution to fine-tuning, since at the same time it seems to preclude a solution to the strong CP problem as discussed in Ref. [27].

(v) The $\mathbb{Z}_{3}$ symmetry could be embedded in a continuous gauge or global group which is broken at some high scale. This is the Lazarides-Shafi mechanism [29], in which the apparent discrete symmetry is a subgroup of the centre of the continuous group. In this case only $\mathrm{U}(1), \mathrm{SU}(3 n)$ (where $n$ is an integer) and $\mathrm{E}_{6}$ are suitable candidates (see for example Ref. [28]). After the electroweak phase transition, one expects only a network of walls bounded by strings to form and then quickly collapse [29].

To summarize, we have shown that the domain wall problem in the NMSSM causes it to be ruled out on cosmological grounds unless we break the $\mathbb{Z}_{3}$ symmetry of the model explicitly. The breaking may be driven by terms which are non-renormalisable and have no direct effect on the low energy theory. However their introduction will in general generate terms which destabilise the hierarchy. In models with "minimal" Kähler potentials, we have shown that there are no non-renormalisable operators which can be added to the superpotential with a coefficient which is simultaneously large enough to solve the cosmological problem and small enough to avoid reintroducing the hierarchy problem. Furthermore, if any of these operators are allowed by the symmetries of the theory at the supergravity scale, then there is no possible symmetry which could prevent the existence of an operator $z N$ in the superpotential whose coefficient must be $\lesssim 10^{-17}$. If we allow mixing between the hidden and visible sectors, the reintroduction of the hierarchy problem can be avoided, and the naturalness problem can be formulated in a way very similar to the $\mu$ problem in the MSSM. However, even here we must arbitrarily select coefficients of certain dangerous operators to be of $\mathcal{O}\left(10^{-9}\right)$ or less once we have arranged for a $\mu$ parameter of a reasonable size, and we have also reintroduced the $\mu$ problem which the model was at least partly designed to solve. Thus we conclude that the parameters in the NMSSM must be very strongly fine tuned if we wish to avoid both the cosmological problems associated with domain walls and the hierarchy problem, and hence that the model is unnatural. 


\section{Acknowledgements}

We would like to thank Graham Ross for encouraging us to undertake this study and for many helpful discussions. We are also grateful to H. Dreiner, U. Ellwanger, E.W. Kolb and M. Rausch de Traubenberg for their criticism and comments, and especially to J. Bagger for discussions concerning destabilising divergences.

\section{References}

[1] For reviews see, H.P. Nilles, Phys. Rep. 110 (1984) 1; H.E. Haber and G.L. Kane, Phys. Rep. 117 (1985) 75.

[2] L. Hall, J. Lykken and S. Weinberg, Phys. Rev. D 27 (1983) 2359; J.E. Kim and H.P. Nilles, Phys. Lett. B 138 (1984) 150; K. Inoue, A. Kakuto and T. Takano, Prog. Theor. Phys. 75 (1986) 664; A.A. Ansel'm and A.A. Johansen, Phys. Lett. B 200 (1988) 331.

[3] G. Giudice and A. Masiero, Phys. Lett. B 206 (1988) 480.

[4] P. Fayet, Nucl. Phys. B 90 (1975) 104; H.-P. Nilles, M. Srednicki and D. Wyler, Phys. Lett. B 120 (1983) 346; J.-M. Frere, D.R.T. Jones and S. Raby, Nucl. Phys. B 222 (1983) 11; J.-P. Derendinger and C.A. Savoy, Nucl. Phys. B 237 (1984) 307;

L. Durand and J.L. Lopez, Phys. Lett. B 217 (1989) 463;

M. Drees, Intern. J. Mod. Phys. A 4 (1989) 3645;

J. Ellis, J. Gunion, H. Haber, L. Roszkowski and F. Zwirner, Phys. Rev. D 39 (1989) 844.

[5] J. Espinosa and M. Quiros, Phys. Lett. B 279 (1992) 92; B 302 (1993) 51;

U. Ellwanger and M. Lindner, Phys. Lett. B 301 (1993) 365;

U. Ellwanger and M. Rausch de Traubenberg, Z. Phys. C 53 (1992) 521;

U. Ellwanger, Phys. Lett. B 303 (1993) 271;

G. Kane, C. Kolda and J. Wells, Phys. Rev. Lett. 70 (1993) 2686;

T. Elliott, S.F. King and P.L. White, Phys. Lett. B 305 (1993) 71; B 314 (1993) 56; P.N. Pandita, Phys. Lett. B 318 (1993) 338; Z. Phys C 59 (1993) 575; T. Elliott, S.F. King and P.L. White, Phys. Rev. D 49 (1994) 2435; U. Ellwanger, M. Rausch de Traubenberg and C.A. Savoy, hep-ph/9502206; F. Franke and H. Fraas, Phys. Lett. B 336 (1994) 415.

[6] B.R. Greene and P.J. Miron, Phys. Lett. B 168 (1986) 226;

R.A. Flores, K. Olive and D. Thomas, Phys. Lett. B 263 (1991) 425;

K. Olive and D. Thomas, Nucl. Phys. B 355 (1991) 192;

S.A. Abel, S. Sarkar and I.B. Whittingham, Nucl. Phys. B 392 (1993) 83.

[7] Ya.B. Zel'dovich, I.Yu. Kobzarev and L.B. Okun, Sov. Phys. JETP 40 (1975) 1.

[8] T.W.B. Kibble, J. Phys. A 9 (1976) 1387; Phys. Rep. 67 (1980) 183.

[9] A. Vilenkin, Phys. Rep. 121 (1985) 263;

A. Vilenkin and E.P.S. Shellard, Cosmic strings and other topological defects (Cambridge Univ. Press, Cambridge, 1994).

[ 10] B. Holdom, Phys. Rev. D 28 (1983) 1419;

B. Rai and G. Senjanovic, Phys. Rev. D 49 (1994) 2729.

[11] J. Ellis, K. Enqvist, D.V. Nanopoulos, K. Olive, M. Quiros and F. Zwimer, Phys. Lett. B 176 (1986) 403.

[12] U. Ellwanger, Phys. Lett. B 133 (1983) 187;

J. Bagger and E. Poppitz, Phys. Rev. Lett. 71 (1993) 2380;

J. Bagger, E. Poppitz and L. Randall, hep-ph/9505244;

V. Jain, Phys. Lett. B 351 (1995) 481.

[13] S.A. Abel and P.L. White, hep-ph/9505241.

[14] U. Ellwanger, M. Rausch de Traubenberg and C.A. Savoy, Phys. Lett. B 315 (1993) 331, hep$\mathrm{ph} / 9502206$; 
Ph. Brax, U. Ellwanger and C.A. Savoy, Phys. Lett. B 347 (1995) 269;

T. Elliott, S.F. King and P.L. White, Phys. Lett. B 351 (1995) 213;

S.F. King and P.L. White, hep-ph/9505326.

[15] H. Hodges, Phys. Rev. D 39 (1989) 3557.

[16] D. Stauffer, Phys. Rep. 54 (1979) 1;

B. Lorenz, I. Orgzall and H.-O. Heuer, J. Phys. A 26 (1993) 4711.

[17] S. Flügge, Practical quantum mechanics (Springer, Berlin, 1971).

[18] L. Kawano, Phys. Rev. D 41 (1990) 1013.

[19] W.H. Press, B.S. Ryden and D.N. Spergel, Astrophys. J. 347 (1989) 590; 357 (1990) 293.

$[20]$ D. Seckel, Inner Space-Outer Space, eds. E.W. Kolb et al. (University of Chicago Press, 1986) p. 367.

[21] L.P. Grischuk and Ya.B. Zeldovich, Sov. Astron. 22 (1978) 125.

[22] J. Ellis, G.B. Gelmini and S. Sarkar, Nucl. Phys. B 373 (1992) 399;

W. Hu and J. Silk, Phys. Rev. Lett. 70 (1993) 2661;

S. Sarkar, Oxford preprint OUTP-95-16P (submitted to Rep. Prog. Phys.).

123] M.H. Reno and D. Seckel, Phys. Rev. D 37 ( 1988) 3441;

S. Dimopoulos, R. Esmailzadeh, L.J. Hall and G.D. Starkman, Nucl. Phys. B 311 (1989) 699.

[24] G.D. Coughlan, W. Fischler, E.W. Kolb, S. Raby and G.G. Ross, Phys. Lett. B 131 (1983) 59.

[25] L. Randall and S. Thomas, Nucl. Phys. B 449 (1995) 229;

D.H. Lyth and E.D. Stewart, Phys. Rev. Lett. 75 (1995) 201.

[26] K.A. Olive, Phys. Rep. 190 (1990) 307;

A. Liddle and D. Lyth, Phys. Rep. 231 (1993) 1.

[27] J. Preskill, S.P. Trivedi, F. Wilczek and M.B. Wise, Nucl. Phys. B 363 (1991) 207.

[28] J.E. Kim, Phys. Rep. 150 (1987) 1.

[29] G. Lazarides and Q. Shafi, Phys. Lett. B 115 (1982) 21;

S.M. Barr, D.B. Reiss and A. Zee, Phys. Lett. B 116 (1982) 227. 